\title{
Uncertainty in Electric Propulsion Erosion Measurements
}

\author{
J. Mackey, ${ }^{1}$ J. Frieman ${ }^{2}$, and D. Ahern ${ }^{3}$ \\ NASA Glenn Research Center, Cleveland, Ohio, 44135, USA \\ and \\ J. Gilland ${ }^{4}$ \\ Ohio Aerospace Institute, Cleveland, Ohio, 44142, USA
}

\begin{abstract}
Uncertainty in erosion rates as measured by different methods is discussed and quantified. The work focuses on case studies from components on the Hall Effect Rocket with Magnetic Shielding (HERMeS) Hall thruster, but the methods can be extended for many electric propulsion applications. The primary method used for evaluating erosion is non-contact profilometry of masked and exposed components. Accurate quantification of the erosion rates of components is critical to determining lifetime and is therefore critical to mission planning purposes.
\end{abstract}

\section{Nomenclature}

$\begin{array}{ll}a & =\text { local erosion depth } \\ e_{i} & =\text { relative uncertainty due to source } i \\ E & =\text { local erosion rate } \\ t & =\text { time of erosion mechanism } \\ U_{i} & =\text { absolute uncertainty due to source } i\end{array}$

\section{Introduction}

For missions beyond low Earth orbit, spacecraft size and mass can be dominated by onboard chemical propulsion systems and propellants that may constitute more than 50 percent of spacecraft mass. This impact can be substantially reduced through the utilization of solar electric propulsion (SEP) due to its significantly higher specific impulse. Studies performed for NASA's Human Exploration and Operations Mission Directorate (HEOMD) and Science Mission Directorate (SMD) have demonstrated that a $40 \mathrm{~kW}$-class SEP capability can be enabling for both near-term and future spacecraft architectures [1]. Since 2012, NASA has been developing a $12.5 \mathrm{~kW}$ Hall-effect thruster electric propulsion string that can serve as the building block for a $40 \mathrm{~kW}$-class SEP capability [2].

The $12.5 \mathrm{~kW}$ Hall thruster system development, led by the NASA Glenn Research Center and the Jet Propulsion Laboratory, began with maturation of the high-power HERMeS (Hall Effect Rocket with Magnetic Shielding) thruster and power processing unit. The technology development work transitioned to Aerojet Rocketdyne following a competitive procurement selection for the Advanced Electric Propulsion System (AEPS) contract. The AEPS contract includes the development, qualification, and multiple flights of $12.5 \mathrm{~kW}$ electric propulsion string deliveries. The AEPS Electric Propulsion (EP) string consists of the Hall thruster, power processing unit (including digital control and interface functionality), xenon flow controller, and associated intra-string harnesses. NASA continues to support the AEPS development leveraging in-house expertise, plasma modeling capability, and world-class test facilities. NASA also executes AEPS and mission risk reduction activities to support the AEPS development and mission application.

Several methods of measuring the erosion rates of components subject to erosion exist and have been demonstrated by many groups. Some methods include direct physical measurement of eroding surfaces with coordinate mapping machine, calipers, micrometers or other appropriate measurement devices. Other techniques involve contact or noncontact profilometry with exposed surfaces measured against masked surfaces or against external reference surfaces

\footnotetext{
${ }^{1}$ Research Engineer, Electric Propulsion Systems Branch, jonathan.a.mackey@nasa.gov, Member AIAA.

${ }^{2}$ Research Engineer, Electric Propulsion Systems Branch, jason.d.frieman@ nasa.gov, Member AIAA.

${ }^{3}$ Research Engineer, Electric Propulsion Systems Branch, drew.ahern@nasa.gov, Member AIAA.

${ }^{4}$ Research Engineer, Electric Propulsion Systems Branch, james.h.gilland@nasa.gov, Member AIAA.
} 
[3-4]. Tracking mass changes of components as they are exposed to erosion is an alternative globally averaged measurement method. Additionally, surface layer activation methods have been used by several groups to make local erosion measurements [10]. Finally, destructive techniques exist such as cross-sectioning exposed components or operating life-tests until an erosion failure of the component. Schedule and cost limitations often make the last option prohibitive, even though it would likely produce the most meaningful results.

Accurate and reliable erosion measurements of plasma exposed components is a critical interest for electric propulsion systems [3,4]. Erosion rates help to determine expected hardware lifetimes and therefore can drive mission planning [5]. In this work, uncertainty of erosion rates will be quantified with a heuristic approach using the traditional method outlined in Refs. [6-9]. The focus will be on the methods and measurements made on components of the HERMeS Hall thruster using non-contact profilometry. However, the presented analysis framework is intended to be sufficiently general to encompass substantially similar alternative measurement approaches. Application of these results to thruster specifications or higher fidelity hardware should be discussed with the authors.

\section{Model of a Masked Erosion Measurement}

The nominal case of an ideal masked component exposed to a general plasma erosion process is outlined in Figure 1. An erosion segment is defined as a period of time when the hardware is deliberately operated at a pre-selected operating condition for an extended time period, with the intent of performing an erosion rate measurement. Masking a component with a sacrificial physical buffer is one method to shield a region of the component from exposure. The masked region can then be used as a datum to measure erosion after the completion of the wear process. The local erosion depth $(a)$ can then be used along with the exposure time $(t)$ to estimate the erosion rate $(E)$.

a)

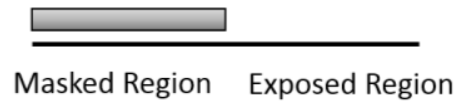

b)

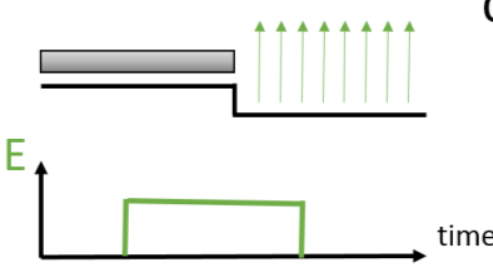

a time c)

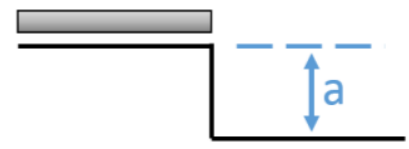

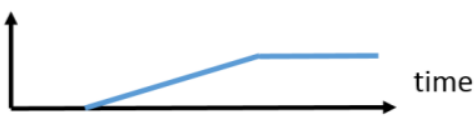

Figure 1: Simplified model of an ideal masked erosion measurement. a) Macroscopically flat, microstructurally smooth, homogeneous component with ideal mask before wear process, b) steady, spatially uniform, directionally invariant erosion process, c) uniform eroded step after erosion process.

In the ideal case the component starts with a perfect macroscopically flat profile, microstructurally smooth surface, and is locally homogeneous. The ideal mask does not interact or alter the substrate and perfectly protects the substrate from erosion or deposition. The idealized erosion process is steady state, spatially uniform, directionally invariant, and contains no back sputter from other sources. The eroded surface is then an ideal step which can be measured with some form of profilometer. This simple model is used to understand the measurement and provides the basic outline of nominal erosion measurement using this technique. The step height as a function of time through the erosion process is shown in Equation 1 as the integration of the desired erosion rate.

$$
a(t)=\int_{t_{0}}^{t} E(\tau) d \tau
$$

For any erosion segment $\mathrm{j}$ the measurable change in step height $\Delta a_{j}$ and segment time $\Delta t_{j}$ are given by Equations 2 and 3 as follows:

$$
\begin{gathered}
\Delta a_{j}=a(t)-a\left(t_{0}\right), \\
\Delta t_{j}=t-t_{0} .
\end{gathered}
$$


The desired erosion rate can them be estimated from the measureable quantities as:

$$
E_{j}=\frac{d a(t)}{d t} \approx \frac{\Delta a_{j}}{\Delta t_{j}}
$$

Equations 1-4 generally apply to a more realistic erosion segment, and serve as the general method for many options of measuring erosion rate. Figure 2 shows a more realistic model of an erosion experiment. The substrate may begin the segment with macroscopically wavy features, may be microstructurally rough, and non-homogeneous (e.g. containing multiple phases or voids). The mask may interact with the substrate, may not perfectly mask the substrate from erosion, and/or may erode and be a source of backsputter material. The erosion process may be transient, spatially varying, directionally varying, and involve backsputter deposition from other sources. The eroded step will not be an ideal step but instead will reflect the several complications of a realistic erosion process.

a)

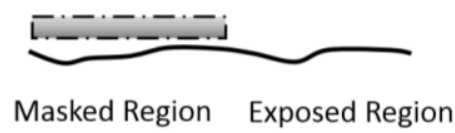

b)
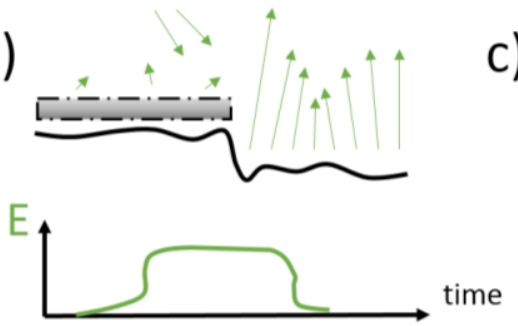

a c)

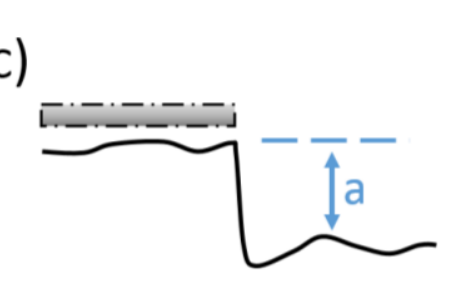

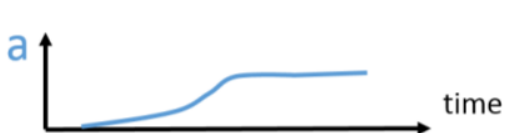

Figure 2: Simplified model of a realistic masked erosion measurement. a) Macroscopically wavy, microscopically rough, non-homogeneous component with realistic mask before wear process, b) transient, spatially varying, directionally varying erosion process, c) non-uniform eroded step after erosion process.

Masks used for the AEPS HERMeS thruster are generally constructed from the same material as the substrate they are protecting. The masks are expected to be sacrificial and their thickness is decided by the expected erosion rate and segment duration to ensure complete coverage until the end of the segment. Masks are attached to their substrate by mechanical fasteners. Figure 3 shows some typical masks used for the AEPS HERMeS thruster. Figure 3a shows the inner front pole cover with radial masks highlighted in red, and the keeper with a slotted mask highlighted in blue. Figure $3 b$ shows a section of the outer front pole cover with radial mask highlighted in green. Masks are setup to provide a masked region surrounded by exposed regions on two opposite sides. 

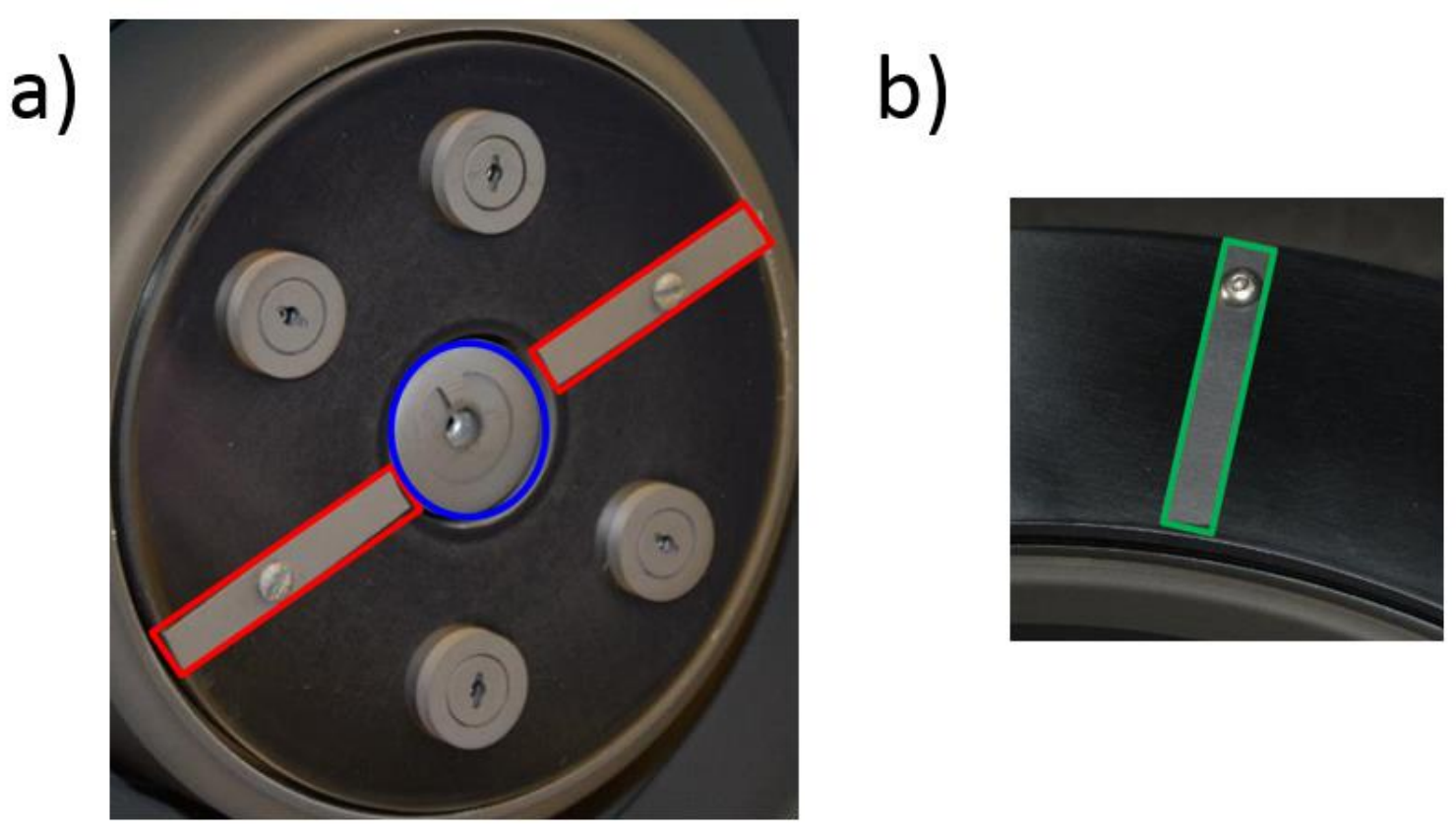

Figure 3: Masks used on Hall thruster components to determine erosion. a) Red masks used on inner front pole covers, blue mask used on keeper. b) Green mask used on outer front pole covers.

Due to edge effects of realistic erosion and backsputter processes and difficulties in performing profilometry on high angle features an exclusion zone is established around the perimeter of every mask. ISO 5436-1 provides a guideline on exclusion and assessment areas around masked regions [11]. Figure 4 shows the ISO 5436-1 type A1 depth measurement standard, which is used to calibrate profilometers. The standard specifies the assessment and exclusion regions in terms of the channel width $\mathrm{W}$, with the low channel region of width $\mathrm{W} / 3$ centered between two high regions of width $2 \mathrm{~W} / 3$. In the case of a masked and eroded region of a thruster component the profile is reversed with the center masked region having the relatively high region centered between two eroded regions. The work to follow uses the recommendations of ISO 5436-1 whenever possible or practical to guide repeatable measurement. 
a)

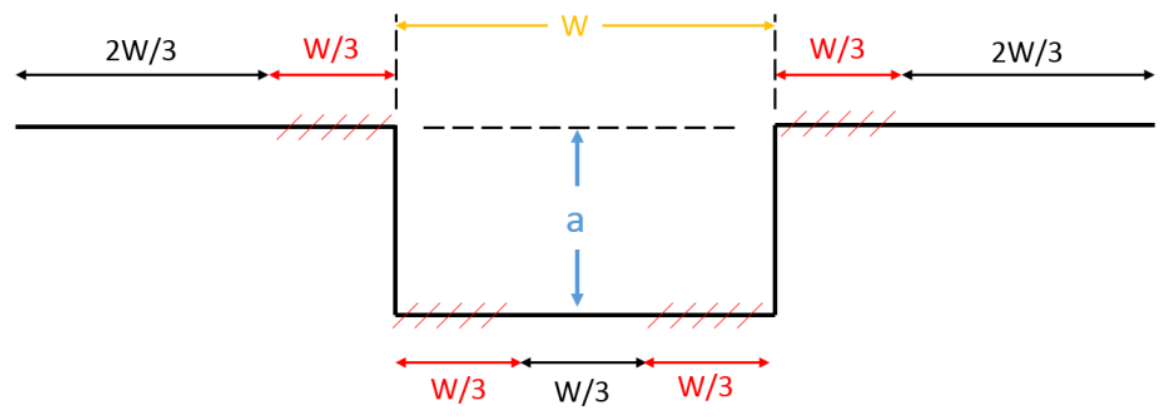

b)

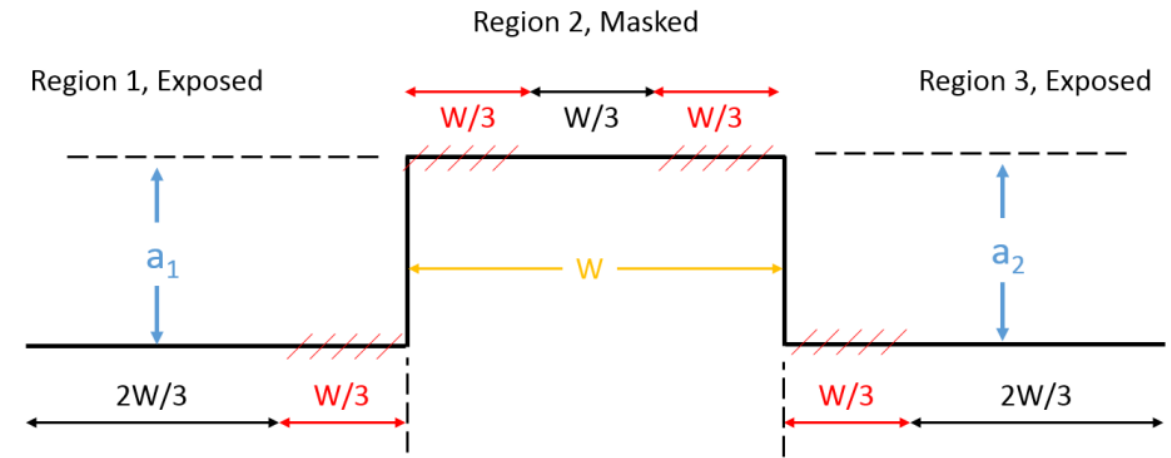

Figure 4: a) ISO 5436-1 Type A1 depth measurement standard recommended assessment areas. Feature width $W$ used to define areas to remove and include in assessment of depth $a$. Regions of $W / 3$ on either side of vertical features are excluded from assessment, remaining regions included in regression. b) Corresponding areas used for a masked erosion measurement.

\section{Uncertainty Quantification}

\section{A. Uncertainty Propagation}

Uncertainty of a dependent variable was approximated using the method outlined by Figliola and Beasley [6] by means of a truncated Taylor series expansion of the measurement formula. Similarly, the uncertainty analysis is as much as possible in accordance with the work of Abernethy et. al [7], Moffat [8], and a more recent NASA Measurement Uncertainty Analysis Principles and Methods Handbook [9] which follows the ISO Guide to the Expression of Uncertainty in Measurement [12]. Absolute uncertainties $U_{x}$ of the independent measurands were accounted for from each independent source of uncertainty whenever possible. The truncated Taylor series approximation of uncertainty propagation of an arbitrary function $y=f(x)$ can be expressed as,

$$
\bar{y} \pm U_{y}=f\left(\bar{x} \pm U_{x}\right) \approx f(\bar{x}) \pm\left.\frac{d f}{d x}\right|_{x=\bar{x}} U_{x} .
$$

Absolute uncertainties were normalized to relative uncertainties for the dependent variable $e_{y_{i}}$, normalization was done with nominal values of the dependent variable. The relative sources were combined using a root sum of square (RSS) type inner-product. The RSS combined relative uncertainty sources lead to an estimated total relative uncertainty. The RSS method provides a suitable combination strategy for statistically independent sources of uncertainty (zero covariance for any two error sources). A more conservative estimate of uncertainty may be to simply arithmetically add the different sources of uncertainty to obtain a total uncertainty, but this generally leads to over estimation of uncertainty [7,9]. The definition of relative uncertainty for some uncertainty source $x$, and the RSS combination of a number of sources is expressed as,

$$
e_{y_{x}}=\left.\frac{1}{\bar{y}} \frac{\partial y}{\partial x}\right|_{x=\bar{x}} U_{x}
$$




$$
e_{\text {Total }}=\sqrt{e_{y_{1}}^{2}+e_{y_{2}}^{2}+e_{y_{3}}^{2}+\cdots} .
$$

In many cases analytic formulation of all sources of uncertainty may become tedious, in which case a method like Monte-Carlo simulation may be employed. For the analysis of this work the sources investigated could be handled analytically, resulting in meaningful closed form terms to be used for system design and improvement.

\section{B. Uncertainty Sources}

A number of factors contribute to the uncertainty of non-contact profilometry based masked erosion measurements. The main sources are outlined in Table 1. Calibration uncertainty is contributed from instrument settings and measurement setup, the details of which are shown in Table 2. Component roughness contributes a source of uncertainty by obscuring the true location of a surface. In practice the roughness of the masked region and the exposed region may be significantly different and are therefore handled individually in Table 1 . The uncertainty is calculated as a 95\% confidence interval of the values recorded in the appropriate assessment area as defined by Figure 4. The roughness uncertainty source is intended to account for surface microscopic roughness of the component but may also account for small voids or multi-phase materials with texture. Component waviness contributes a large component of uncertainty and results from the simple assumption involved in Figure 4, that the surface is nominally flat. Strain induced by mechanical fasteners, polishing and handling processes, and geometric variation all contribute to the component waviness. Deviation of the surface from a flat plane in the assessment regions leads to the uncertainty and two main methods of quantifying the uncertainty have been used.

Table 1: Sources of relative uncertainty: overbar represents nominal value.

\begin{tabular}{l|c|c|l}
\hline \multicolumn{1}{c}{ Source } & Relative Uncertainty & $\begin{array}{c}\text { Parameters } \\
\text { of Interest }\end{array}$ & \multicolumn{1}{c}{ Parameter Description } \\
\hline Calibration uncertainty & $e_{c a l}=\frac{U_{c a l}}{\bar{a}}$ & $U_{c a l}$ & $\begin{array}{l}\text { Calibration uncertainty, see table } \\
2 .\end{array}$ \\
\hline $\begin{array}{l}\text { Component roughness, } \\
\text { masked region }\end{array}$ & $e_{\text {masked }}=\frac{t_{99 \%, v} s_{\text {masked }}}{\bar{a} \sqrt{n_{\text {masked }}}}$ & $\begin{array}{l}S_{\text {masked }} \\
n_{\text {masked }}\end{array}$ & $\begin{array}{l}\text { Masked standard deviation, } \\
\text { Masked measurement count. }\end{array}$ \\
\hline $\begin{array}{l}\text { Component roughness, } \\
\text { exposed region }\end{array}$ & $e_{\text {exposed }}=\frac{t_{99 \%, v} s_{\text {exposed }}}{\bar{a} \sqrt{n_{\text {exposed }}}}$ & $\begin{array}{l}s_{\text {exposed }} \\
n_{\text {exposed }}\end{array}$ & $\begin{array}{l}\text { Exposed standard deviation, } \\
\text { Exposed measurement count. }\end{array}$ \\
\hline $\begin{array}{l}\text { Component waviness } \\
\text { Segment time off-point }\end{array}$ & $e_{\text {wavy }}=\frac{U_{\text {wavy }}}{\bar{a}}$ & $U_{\text {wavy }}$ & $\begin{array}{l}\text { Deviation of surface from straight } \\
\text { line background correction. }\end{array}$ \\
\hline
\end{tabular}

Figure 5 demonstrates the concept of a component waviness source of uncertainty. The dashed blue lines represent the nominal flat features from which the hypothetical erosion step is measured. In this example due to component waviness the step height measured varies with the distance from the step $\ell_{i}$. The variation is on a macroscopic distance where the averaging length of each step measurement may be on the order of the $1 \mathrm{~mm}$ sampling length. The length scales involved are distinct from the microscopic roughness terms already discussed in Table 1 . As demonstrated in Figure 5 a range of step heights may be calculated at different distances $\ell_{i}$. One method to estimate the waviness uncertainty is to collect a set of step heights at a fixed sampling length over a range of distances from the step, then to investigate measures of spread of the data. An alternative method is to investigate component flatness before and after an erosion process and asses the contribution of uncertainty as a result. The challenge with this alternative method is the implicit assumption that the flatness of the component does not change. This assumption is likely invalid due to thermal cycling of components, pressure cycling of components, mechanical attachment and removal of components, and other unknown sources. As a result for the work to follow component waviness is calculated as the range of the study demonstrated in Figure 5. 


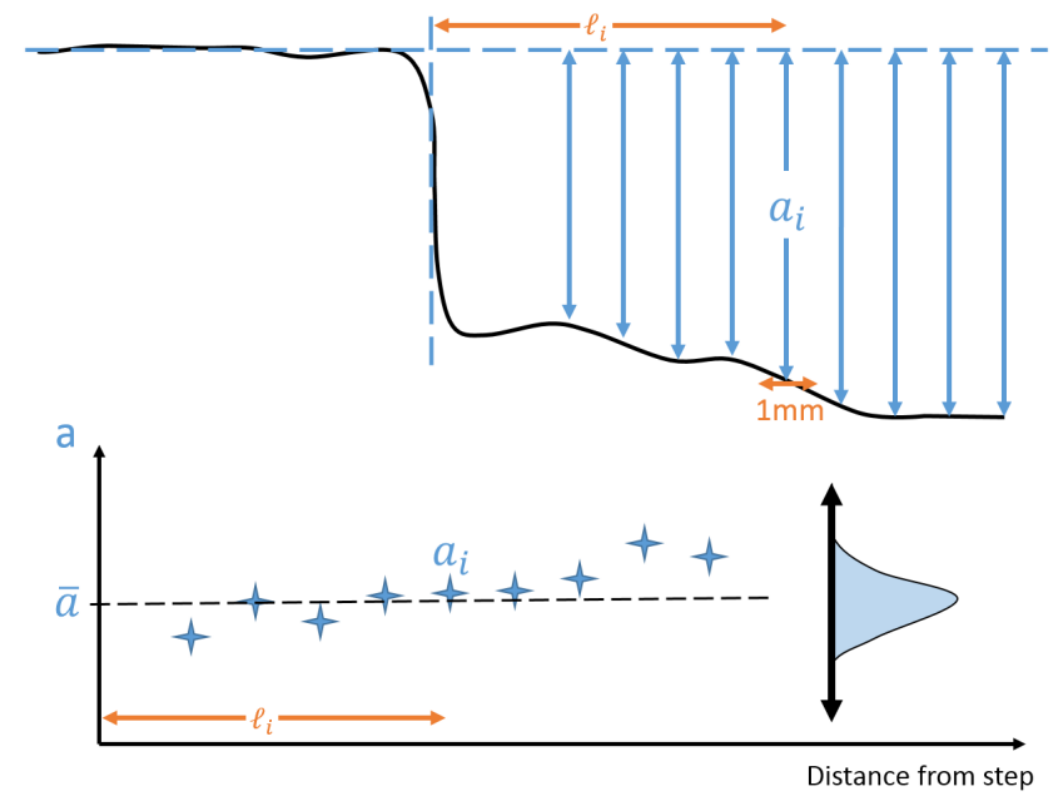

Figure 5: Example of component waviness uncertainty. Erosion step height measurements calculated over a range of distances from the erosion step.

The last significant source of uncertainty identified by this work is the segment time off-point. During any erosion segment, the thruster should ideally be operated at only one condition leading to one uniform erosion rate. In practice this is not practical due to programmatic/schedule needs and fundamental changes in the thruster. In many cases erosion segments need to be sufficiently long to ensure accurate rate measurement, and during long segments thrusters may need to be operated at off-point operating conditions, or factors out of control of the operator may cause erosion rates to change. Figure 6 sketches the simplest estimate of accounting for the off-point uncertainty. The assumption is that the nominal erosion rate to be determined is $E_{1}$ and the short off-point rate is $E_{2}$. The resulting step of the actual segment tested is $\Delta a$ when the nominal step would have been $\Delta a^{\prime}$. The difference between the actual step and nominal step can be estimated using equation 8 .

$$
\Delta a-\Delta a^{\prime}=\int\left[E_{2}(\tau)-E_{1}(\tau)\right] d \tau \approx\left[E_{2}-E_{1}\right] \Delta t_{o f f-p o i n t}
$$

If the on-point and off-point erosion rates can be estimated by some means then the on-point erosion rate can be estimated with equation 9 , and the uncertainty due to a number of off-point rates can be estimated by equation 10 . The intention of the estimation is to capture the likely uncertainty resulting from operating off-point, rather than to calculate precisely the actual nominal rate.

$$
\begin{gathered}
E_{1}=\frac{\Delta a^{\prime}}{\Delta t} \approx \frac{\Delta a-\left[E_{2}-E_{1}\right] \Delta t_{\text {off-point }}}{\Delta t} \\
e_{\text {time }}=\sum_{i} \frac{\left[E_{i}-E_{1}\right] \Delta t_{\text {off-point }}}{\bar{a}}
\end{gathered}
$$



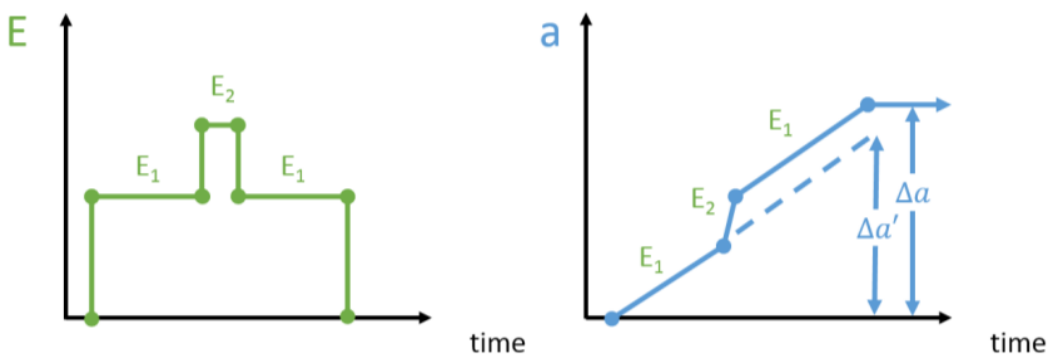

Figure 6: Example of time off-point uncertainty. Operating at an off-point condition for a short duration can lead to uncertainty in the calculated erosion step.

The final source of uncertainty considered in this work is the calibration uncertainty associated with the profilometer itself. Table 2 highlights the component calibration uncertainty factors studied in this work. The factors of interest are sensitive to each instrument used to perform the measurements. This work used a Nanovea ST400C (vendor details included only for completeness, not intended to serve as an endorsement) non-contact chromatic confocal profilometer for making step measurements. The factors listed in table 2 are particular to settings and parameters for the applicable system. The remaining sources of uncertainty listed in Table 1 are likely consistent for other measurement instruments and are instead a factor of the masked method in general. The first calibration/instrument factor is step size, which is a measure of the distance between adjacent measurement locations. The next factor is the number of points taken and averaged at any single physical location. The acquisition rate is the sampling frequency of the collection optics of the profilometer. The brightness is the illumination brightness intensity used for the optics. The focus height is the nominal distance between the optics and the surface feature, depending on focal range of optics this range may be restricted to specific ranges. The work to follow uses a nominally $3 \mathrm{~mm}$ focal distance optic. Finally the off-nominal tilt is the angle off perpendicular between the collection optic and the surface feature. The parameter ranges used in Table 2 were based on operator experience, physical limitations, or typical values generally used in testing.

Table 2: Factors of calibration/instrument settings uncertainty.

\begin{tabular}{ll|l}
\hline \multicolumn{1}{c}{ Factor } & Factor Range & \multicolumn{1}{c}{ Factor Description } \\
\hline Step Size & $1-15 \mu \mathrm{m}$ & $\begin{array}{l}\text { Physical distance between single measurement } \\
\text { locations. }\end{array}$ \\
\hline Averaging & $1-20$ points & $\begin{array}{l}\text { Repeated measurement averaging performed } \\
\text { at a single location. }\end{array}$ \\
\hline Acquisition Rate & $200-1000 \mathrm{~Hz}$ & \begin{tabular}{l} 
Sampling frequency of the collection optics. \\
\hline Brightness
\end{tabular} \\
\hline Focus Height & $25-100 \%$ & Illumination brightness intensity of the optics. \\
\hline Off-Nominal Tilt & $\pm 44-2047 \mu \mathrm{m}$ & $\begin{array}{l}\text { Nominal distance between optics and surface } \\
\text { feature. }\end{array}$ \\
\hline
\end{tabular}

\section{Uncertainty Calculation}

The final erosion rate is estimated using Equation 4 averaged across the steps of regions 1 and $3 \tilde{a}$ and the segment nominal operating time $\Delta t$. Averaging across both sides of the step tends to average out azimuthal asymmetry which has been found to exist on some of the components studied in this work. The total uncertainty of the erosion rate is estimated from Equation 11 as:

$$
U_{E}=\frac{\tilde{a} e_{\text {masked }}+\tilde{a} e_{\text {exposed }}+\sqrt{\left(\tilde{a} e_{c a l}\right)^{2}+\left(\tilde{a} e_{\text {wavy }}\right)^{2}+\left(\tilde{a} e_{t i m e}\right)^{2}}}{\Delta t} .
$$

The uncertainty due to instrument settings, component waviness, and off-point segment time are assumed to be uncorrelated. Whereas the uncertainty due to roughness of the masked and exposed areas are treated as perfectly correlated, as the measurement of a step depends on determining the location of both surfaces which are each obscured by roughness. 


\section{Uncertainty Analysis Case Studies}

The method of measuring erosion rate uncertainty is demonstrated using two case studies. The case studies were both taken on graphite inner front pole covers of a HERMeS thruster. The red highlighted mask of Figure 3a is the mask of interest for both case studies. The two cases were measured after operating the thruster at two different operating points for two different segment times. The influence of thruster operating points on erosion is beyond the scope of this work, so the operating conditions will not be discussed herein. Instead, the two cases will be referred to by their nominal segment operating times which were 1015 hours and 250 hours. The majority of the examples will be generated from the 1015 hour example, as it represents the longest segment taken to date and therefore provides the best case analysis for determining erosion rate. In practice operating a series of 1015 hour single erosion segments can be prohibitive to schedule and budget, and therefore a shorter duration is more likely to be used for most work. The 250 hour case will be summarized with final values to serve as a contrast for a more practical case.

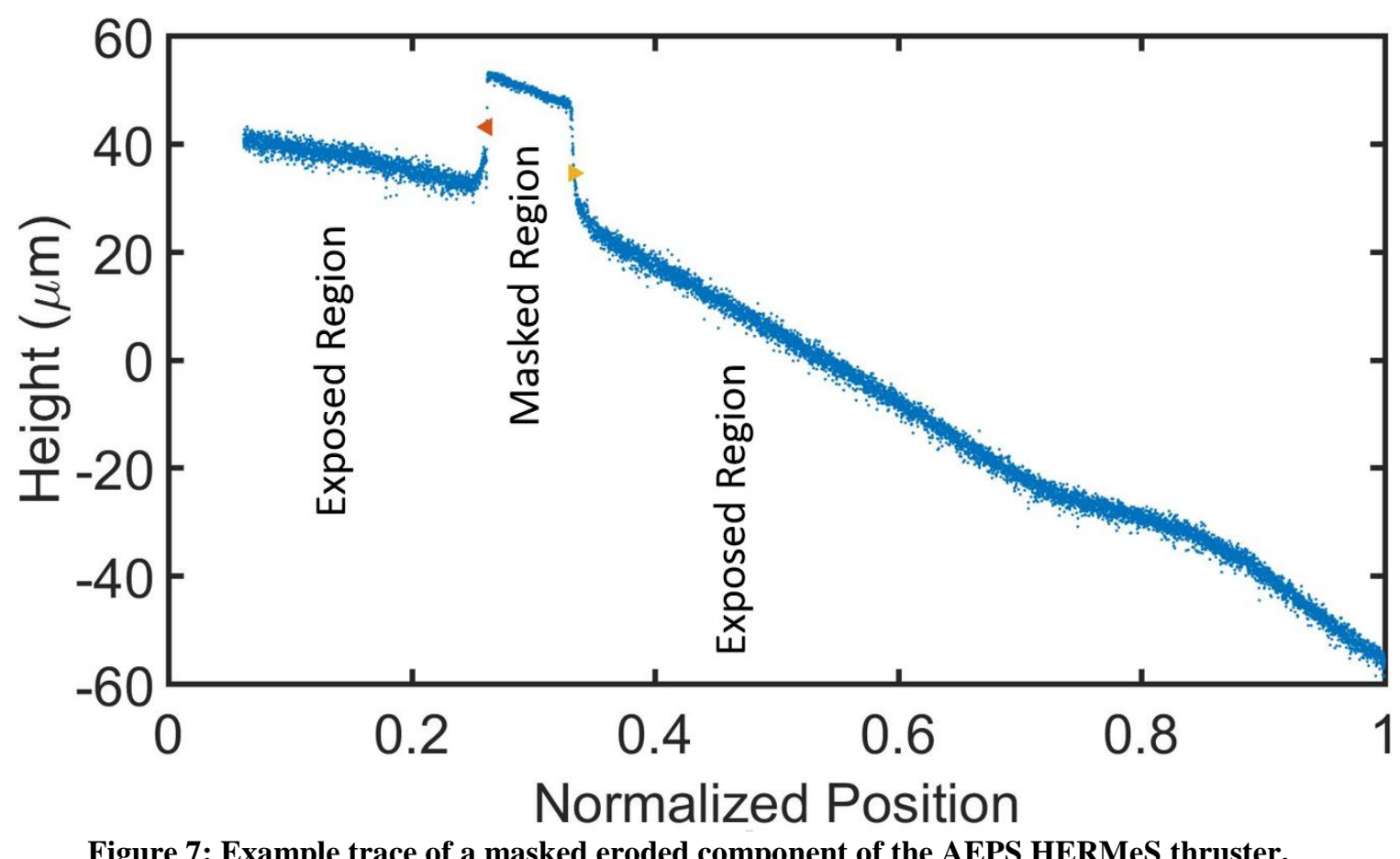

Figure 7: Example trace of a masked eroded component of the AEPS HERMeS thruster. 


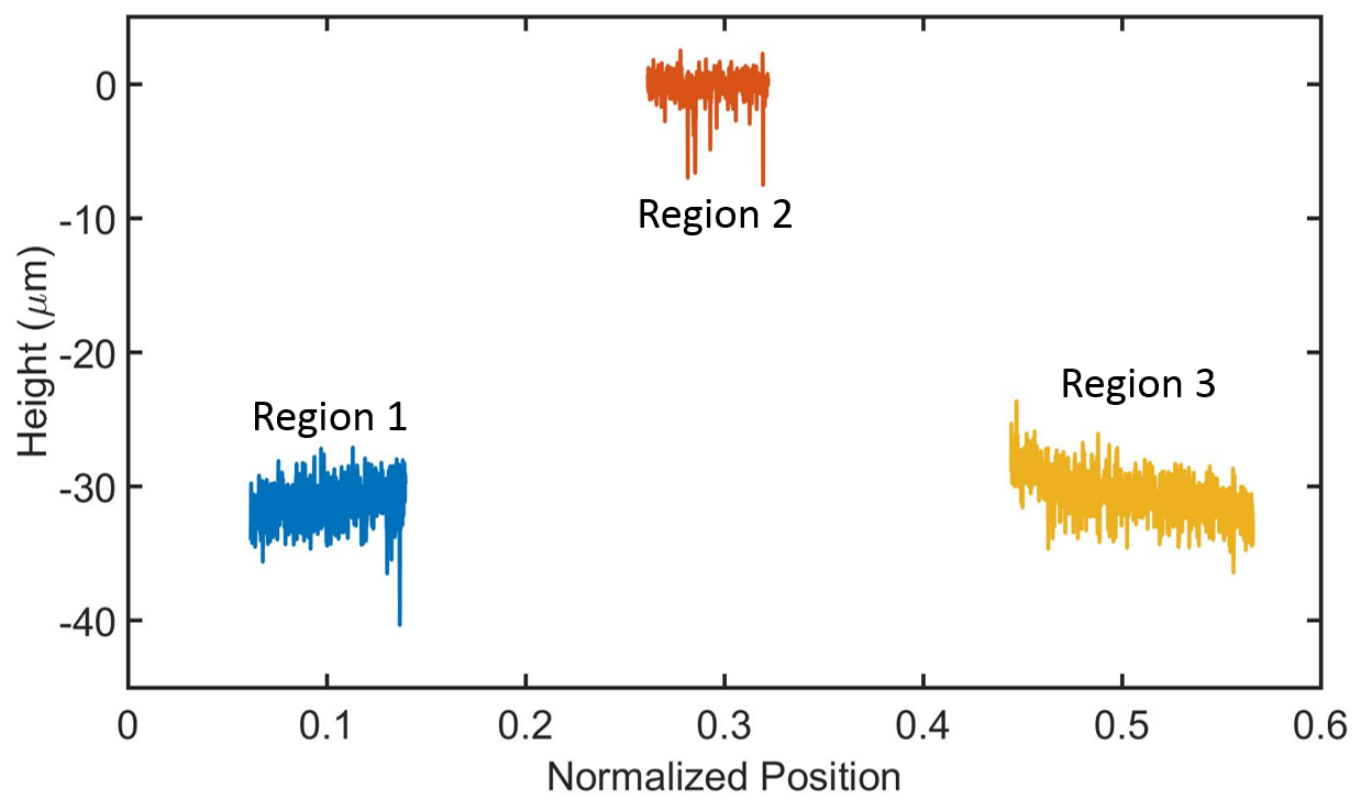

Figure 8: Example erosion rate measurement regions of interest of trace shown in Figure 7. Exposed regions are 1 and 3 and masked region is 2.

A set of profilometer traces were collected across different radii of the inner front pole covers for both the 1015 and 250 hour cases. Only a single trace was selected from each dataset to serve as the demonstration case for the work to follow; the full analysis of all radius can be found in Reference 3. The raw trace of the 1015 hour case is shown in Figure 7. The masked region can be clearly seen as the feature protruding above the background. The location of the masked region is identified automatically using a peak finding routine on the first finite difference of the trace. The location of the mask edges are marked in the figure by two triangles, the width between the edges is used to extract the excluded and assessment regions. The two exposed assessment regions are then background corrected to the masked assessment region. Figure 8 shows the final extracted and background corrected trace used for the erosion rate measurement. The nominal erosion rate can then be calculated using the average step height between exposed regions 1 and 3 and the segment operating time. In this case the average step height is $30.82 \mu \mathrm{m}$ leading to an erosion rate of $30.36 \mu \mathrm{m} / \mathrm{khr}$ over the 1015 hour segment. For the 250 hour case the same method was used, but the trace is not shown. The resulting average step height was $12.61 \mu \mathrm{m}$ leading to an erosion rate of $50.43 \mu \mathrm{m} / \mathrm{khr}$ over the 250 hour segment. The work to follow provides a conservative estimate for the total uncertainty of the calculated erosion rates.

To quantify the calibration/instrument settings uncertainty two NIST traceable depth measurement standards were scanned with the range of parameters of Table 2 . The depth measurement standards were nominally $10 \mu \mathrm{m}$ and 50 $\mu \mathrm{m}$ quartz standards. The step height of each scan in Table 3 was calculated using the same methods established herein for measuring erosion rate, then the deviation of the measured step height from the calibrated standard height was calculated. Table 3 includes the 23 cases investigated along with the calculated deviations from the standard step height for the $50 \mu \mathrm{m}$ standard. Values in Table 3 highlighted in black represent the nominal case for each setting parameter, values in red highlight off-nominal parameter settings. The ranges of parameters investigated were selected to represent values that might be realistically used for erosion measurements, so the range of deviations represent the maximum likely uncertainty. The deviations for the study span $+0.34 \mu \mathrm{m}$ to $-0.77 \mu \mathrm{m}$. Similar studies were completed with the $10 \mu \mathrm{m}$ nominal standard and for alternative $20 \mathrm{~mm}$ optics. The results of the study shown capture the general behavior, therefore the other studies have been omitted. The calibration/instrument setting uncertainty has been estimated using two standard deviations of the deviations of the results. The calibration/instrument settings uncertainty is estimated to be $U_{c a l}=0.54 \mu \mathrm{m}$ and is independent of scan so the same value is used for both case studies.

The study presented in Table 3 may contain useful correlations for operating the profilometer in a way to minimize measurement uncertainty. For a thorough study, correlations should be investigated across multiple depth measurement standards and multiple optics assemblies. A future work may address some of the correlations and behaviors of the individual parameters. Only one set of trends are highlighted herein because they are likely applicable across many profilometer based measurements. Figure 9 shows details of the probe tilt study performed on the $50 \mu \mathrm{m}$ nominal depth standard. The angles between the optics assembly and the surface of the standard were measured and varied. The probe was first aligned to $90^{\circ}$ between optics assembly and the standard in the direction parallel to the 
step feature and $90^{\circ}$ between optics assembly and the standard in the direction perpendicular to the step feature. The trace was then collected and the deviation was calculated for the case of $0^{\circ}$ tilt against both parallel and perpendicular orientations of the depth standard. One angle was tilted at a time, either the parallel or perpendicular orientation of the step feature, and is shown in Figure 9. Angles up to $\pm 15^{\circ}$ were investigated. For angles of tilt less the $5^{\circ}$ in either direction, the deviation is within the calibration/instrument settings uncertainty of $0.54 \mu \mathrm{m}$. For tilt angles larger than $\pm 5^{\circ}$ in either direction a significant new source of uncertainty is introduced. In practice erosion components are fairly flat and the nominal angle between component and optics assembly are likely within the $\pm 5^{\circ}$ range.

Table 3: Calibration/instrument settings uncertainty.

\begin{tabular}{|c|c|c|c|c|c|}
\hline $\begin{array}{c}\text { Step Size } \\
(\mu \mathrm{m})\end{array}$ & $\begin{array}{c}\text { Averaging } \\
\text { (Count) }\end{array}$ & $\begin{array}{l}\text { Rate } \\
(\mathbf{H z})\end{array}$ & $\begin{array}{c}\text { Brightness } \\
(\%)\end{array}$ & $\begin{array}{c}\text { Tilt } \\
\text { (Deg) }\end{array}$ & $\begin{array}{c}\text { Deviation } \\
(\mu \mathrm{m})\end{array}$ \\
\hline 1 & 16 & 200 & 100 & 0 & -0.521 \\
\hline 5 & 16 & 200 & 100 & 0 & -0.505 \\
\hline 10 & 16 & 200 & 100 & 0 & -0.481 \\
\hline 15 & 16 & 200 & 100 & 0 & -0.768 \\
\hline 1 & 4 & 200 & 100 & 0 & -0.528 \\
\hline 1 & 8 & 200 & 100 & 0 & -0.648 \\
\hline 1 & 12 & 200 & 100 & 0 & -0.556 \\
\hline 1 & 20 & 200 & 100 & 0 & -0.496 \\
\hline 1 & 16 & 400 & 100 & 0 & -0.504 \\
\hline 1 & 16 & 1000 & 100 & 0 & -0.584 \\
\hline 1 & 16 & 200 & 25 & 0 & -0.550 \\
\hline 1 & 16 & 200 & 50 & 0 & -0.627 \\
\hline 1 & 16 & 200 & 75 & 0 & -0.655 \\
\hline 1 & 16 & 200 & 100 & -5 & -0.127 \\
\hline 1 & 16 & 200 & 100 & -4 & -0.409 \\
\hline 1 & 16 & 200 & 100 & -3 & -0.097 \\
\hline 1 & 16 & 200 & 100 & -2 & 0.167 \\
\hline 1 & 16 & 200 & 100 & -1 & 0.348 \\
\hline 1 & 16 & 200 & 100 & 1 & -0.106 \\
\hline 1 & 16 & 200 & 100 & 2 & -0.442 \\
\hline 1 & 16 & 200 & 100 & 3 & -0.291 \\
\hline 1 & 16 & 200 & 100 & 4 & -0.149 \\
\hline 1 & 16 & 200 & 100 & 5 & -0.378 \\
\hline
\end{tabular}

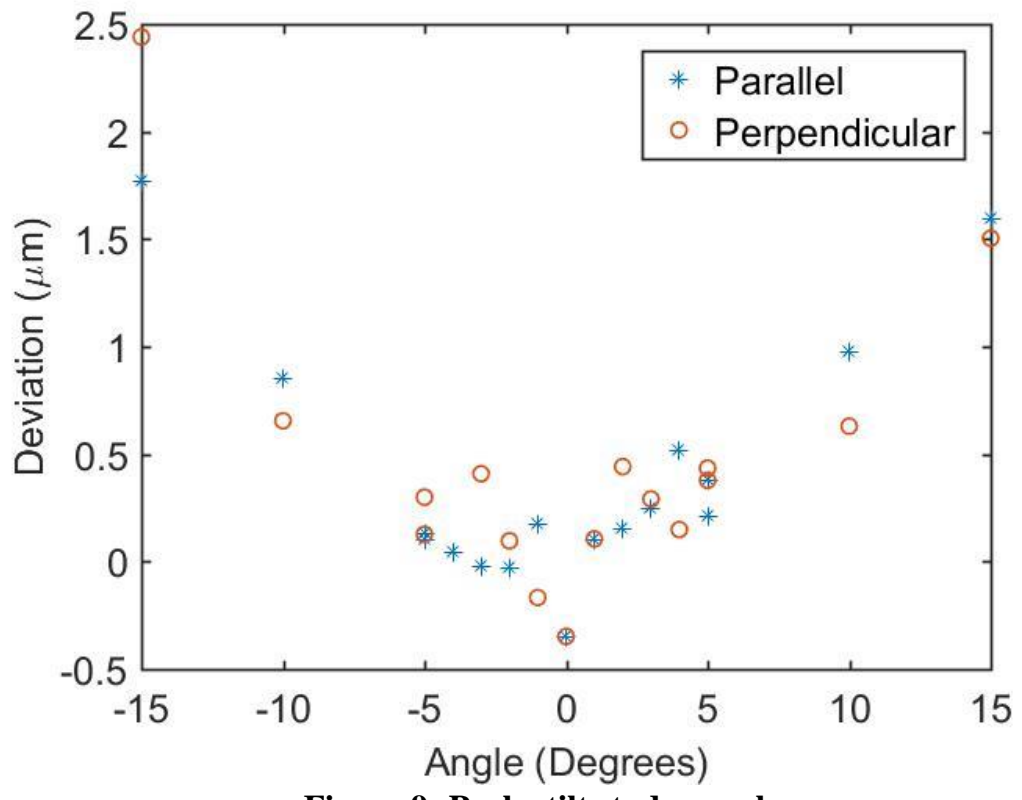

Figure 9: Probe tilt study graphs 
To quantify the roughness uncertainness of the masked and exposed regions, histograms were generated for each region. Figure 10a shows the histogram of the exposed region 1 after background correcting to the masked region 2. Figure 10b shows exposed region 3 after background correcting to the masked region 2. Figure 10c shows masked region 2 after background correcting to itself. Figure 10d shows exposed region 1 after background correcting to itself. In general the exposed regions have a range on the order of $10 \mu \mathrm{m}$ if outliers are excluded, and $16 \mu \mathrm{m}$ otherwise. Scanning electron microscopy of the components reveals that the components generally contain pores up to $27 \mu \mathrm{m}$ in ferret diameter and unknown depth. The outliers seen in the scans suggest that the pores may be on the order of $10 \mu \mathrm{m}$ deep. The masked region has a range on the order of $4 \mu \mathrm{m}$ if outliers are excluded, and $12 \mu \mathrm{m}$ otherwise. For this work the components were hand polished before erosion to provide a smooth reference surface of the masked region. The tighter histogram of the masked region is consistent with the polishing; note that polishing does not remove pores so the outliers remain in the dataset. Masked and exposed regions of components were also scanned in a Michelson interferometer style profilometer to provide a higher fidelity view of the surface roughness. Similar histograms were obtained using the Michelson interferometer, indicating that the histograms are in fact an indication of surface roughness and not simple instrument noise of the Nanovea profilometer.

The histograms of the three regions are analyzed independently on every scan to determine the exposed roughness and masked roughness uncertainty. For the 1015 hour study the masked roughness uncertainty was $0.08 \mu \mathrm{m}$ and the exposed roughness was $0.10 \mu \mathrm{m}$. For the 250 hour study the masked roughness uncertainty was $0.12 \mu \mathrm{m}$ and the exposed roughness was $0.15 \mu \mathrm{m}$.
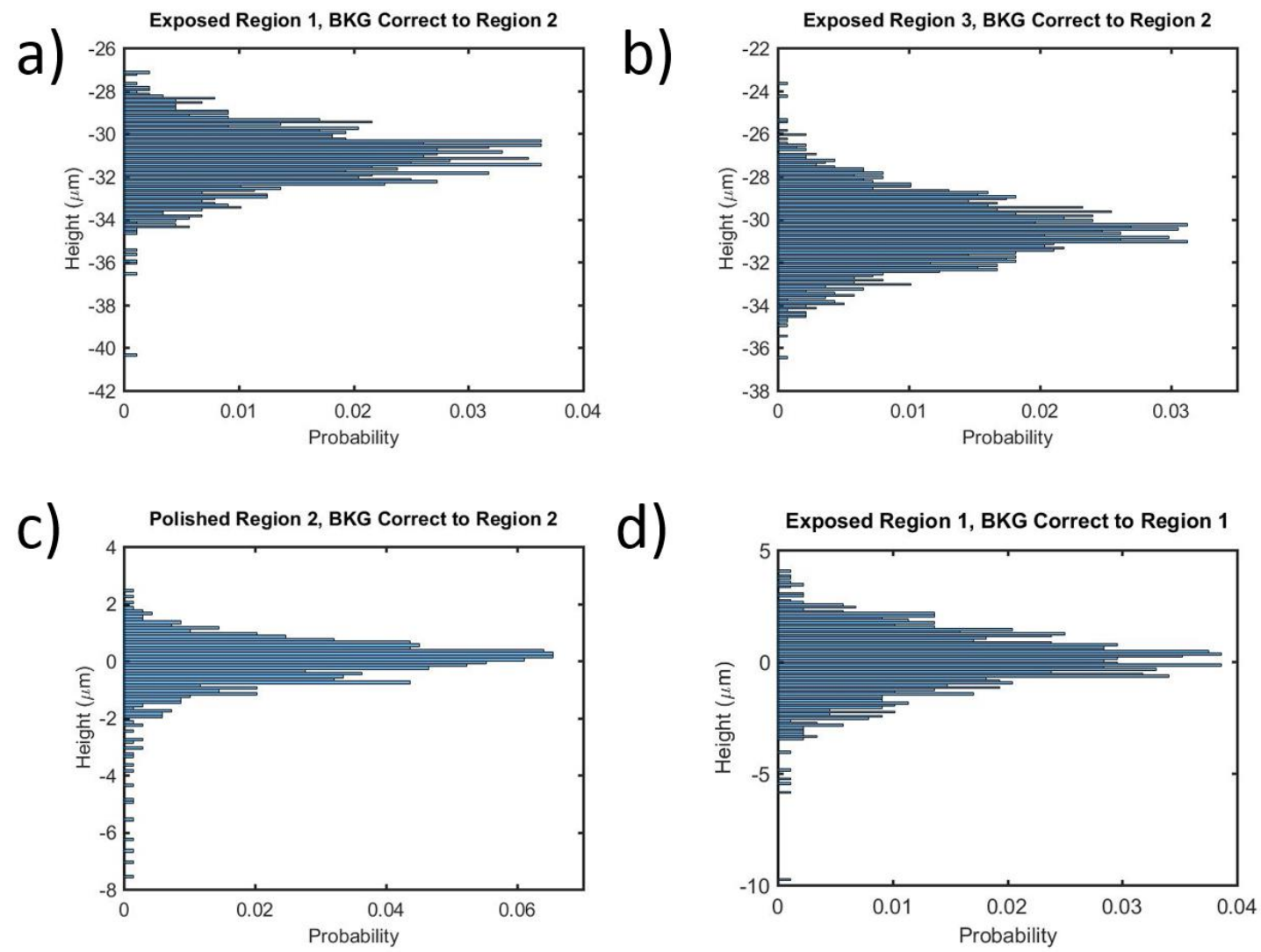

Figure 10: Histograms of regions of interest shown in Figure 7. a) Exposed region 1 background corrected to masked region 2, b) exposed region 3 background corrected to masked region 2, c) masked region 2 corrected to itself, d) exposed region 1 corrected to itself.

To quantify the waviness uncertainty the exposed assessment regions 1 and 3 were divided into 25 sub regions, which were then evaluated for step height relative to the masked region 2 . The step height of each sub region is shown in Figure 11 as a function of the distance from the step edge. Trends in the plots, as shown in Figure 11, suggest macroscopic waviness in the assessment regions. To serve as a conservative estimate of the waviness half the range of the values calculated in the 25 sub regions is used as the uncertainty. Then the maximum uncertainty between 
exposed region 1 and region 3 is used as the final value. For the 1015 hour case study the waviness uncertainty was $2.26 \mu \mathrm{m}$. For the 250 hour case study the waviness uncertainty was $1.73 \mu \mathrm{m}$. Using the range of the sub regions as the uncertainty is fairly conservative and sensitive to outliers, but the method was established by analyzing samples that had detailed pre/post erosion scans. Samples with pre/post data were evaluated to help determine the initial waviness of components and possible uncertainty introduced from straight line fitting. The difficulty of using pre/post data and using non-linear background fitting is that it assumes the components do not change shape during testing, which is likely a poor assumption. To account for this difficulty a more conservative approach of calculating sub region ranges is used.
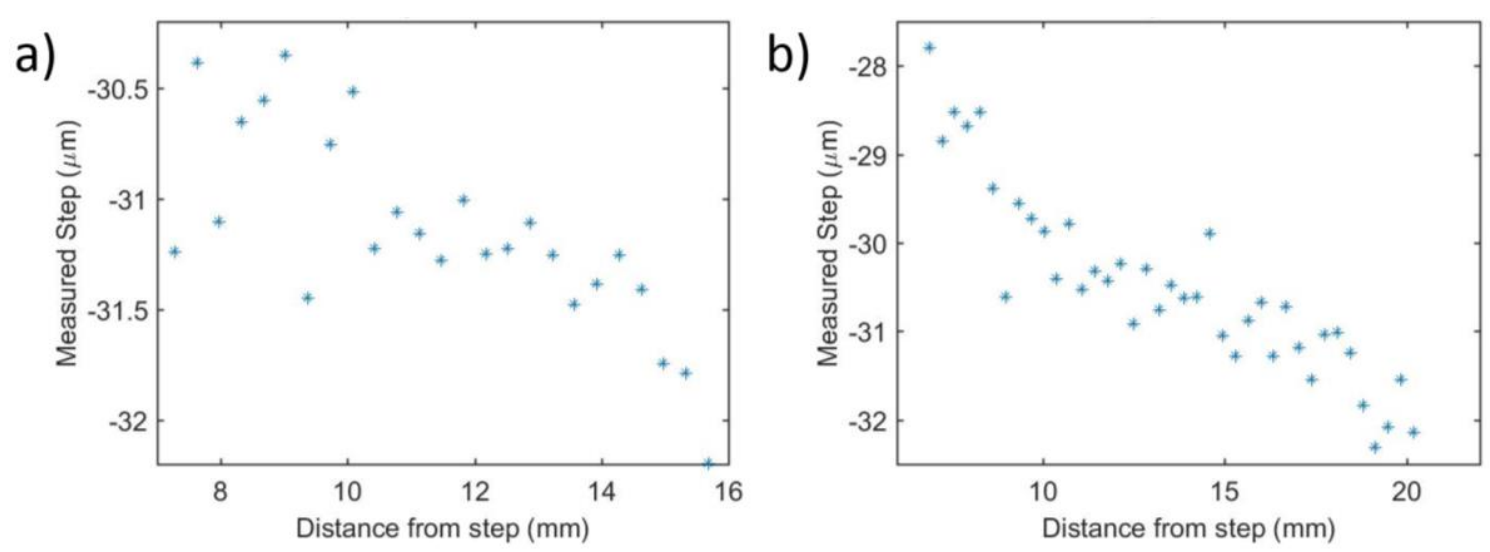

Figure 11: Calculation of waviness uncertainty using method I. a) Steps taken from several locations within exposed region 1, b) steps taken from several locations within exposed region 3.

To quantify the time off-point uncertainty the 1015 hour test segment was evaluated for condition operating time. Six primary operating points were identified and the total operating time at each condition was accumulated. The erosion rate at each condition was estimated using prior experience or modeling. Using equations 8,9 , and 10 the assumed uncertainty due to off-point operation of the 1015 hour case study was found to be $0.63 \mu \mathrm{m}$. The uncertainty value assumes well known operating times, known erosion rates, and ideal erosion behavior of the components. To provide a level of conservatism to the analysis the $0.63 \mu \mathrm{m}$ uncertainty value is used consistently for all shorter segments rather than attempting to calculate off-point operating for a short test sequence. It is likely that a shorter test segment will have a smaller off-point uncertainty, but using the fixed value helps to cover some of the assumptions involved in the calculation.

Table 4: Time at off-point testing conditions for an erosion segment nominally targeting condition 5.

\begin{tabular}{cccc}
\hline $\begin{array}{c}\text { Thruster } \\
\text { Condition }\end{array}$ & $\begin{array}{c}\text { Estimated Erosion } \\
\text { Rate }(\boldsymbol{\mu m} \mathbf{m h h})\end{array}$ & $\begin{array}{c}\text { Time } \\
(\mathbf{h r})\end{array}$ & $\begin{array}{c}\text { Accumulated } \\
\text { Uncertainty }(\boldsymbol{\mu m})\end{array}$ \\
\hline $\mathbf{1}$ & 60 & 4.3 & 0.17 \\
\hline $\mathbf{2}$ & 60 & 5.3 & 0.20 \\
\hline $\mathbf{3}$ & 40 & 6.1 & 0.12 \\
\hline $\mathbf{4}$ & 30 & 6.1 & 0.05 \\
\hline $\mathbf{5 , \text { Nominal }}$ & 21 & 1015.0 & 0.00 \\
\hline $\mathbf{6}$ & 40 & 4.6 & 0.09 \\
\hline
\end{tabular}

The final erosion rate measurements and uncertainty for the 1015 hour case study are summarized in Table 5. Given the significant segment test time the erosion rate can be conservatively estimated at $30.36 \mu \mathrm{m} / \mathrm{khr}$ with an uncertainty of $2.54 \mu \mathrm{m} / \mathrm{khr}$ ( $8.4 \%$ of measured value). For the shorter 250 hour case study the erosion rate is 50.43 $\mu \mathrm{m} / \mathrm{khr}$ with an uncertainty of $14.85 \mu \mathrm{m} / \mathrm{khr}$ ( $29.4 \%$ of measured value). The influence of segment time is apparent when considering the relative uncertainty of the two cases, and the results are amplified for short test sequences at relatively low erosion rates. 
Table 5: Values for 1015 hour case study.

\begin{tabular}{lcc}
\hline \multicolumn{1}{c}{ Parameter } & Term & Value \\
\hline Average step & $\tilde{a}$ & $30.82 \mu \mathrm{m}$ \\
\hline Calibration uncertainty & $U_{\text {cal }}$ & $0.54 \mu \mathrm{m}$ \\
\hline $\begin{array}{l}\text { Component roughness, } \\
\text { masked region }\end{array}$ & $\frac{t_{99 \%, s_{\text {mask }}}}{\sqrt{n_{\text {mask }}}}$ & $0.08 \mu \mathrm{m}$ \\
\hline $\begin{array}{l}\text { Component roughness, } \\
\text { exposed region }\end{array}$ & $\frac{t_{99 \%, v} s_{\text {exposed }}}{\sqrt{n_{\text {exposed }}}}$ & $0.10 \mu \mathrm{m}$ \\
\hline Component waviness & $U_{\text {wavy }}$ & $2.26 \mu \mathrm{m}$ \\
\hline Segment time off-point & $U_{\text {time }}$ & $0.63 \mu \mathrm{m}$ \\
\hline Erosion rate & $E$ & $30.36 \frac{\mu \mathrm{m}}{\mathrm{khr}}$ \\
\hline Total rate uncertainty & $U_{E}$ & $2.54 \frac{\mu \mathrm{m}}{\mathrm{khr}}$ \\
\hline
\end{tabular}

Table 6: Values for 250 hour case study.

\begin{tabular}{l|c|c|}
\hline \multicolumn{1}{c}{ Parameter } & Term & Value \\
\hline Average step & $\tilde{a}$ & $12.61 \mu \mathrm{m}$ \\
\hline Calibration uncertainty & $U_{\text {cal }}$ & $0.54 \mu \mathrm{m}$ \\
\hline $\begin{array}{l}\text { Component roughness, } \\
\text { masked region }\end{array}$ & $\frac{t_{99 \%, v} s_{\text {mask }}}{\sqrt{n_{\text {mask }}}}$ & $0.12 \mu \mathrm{m}$ \\
\hline $\begin{array}{l}\text { Component roughness, } \\
\text { exposed region }\end{array}$ & $\frac{t_{99 \%, v} s_{\text {exposed }}}{\sqrt{n_{\text {exposed }}}}$ & $0.15 \mu \mathrm{m}$ \\
\hline Component waviness & $U_{\text {wavy }}$ & $1.73 \mu \mathrm{m}$ \\
\hline Component waviness & $U_{\text {wavy }}$ & $3.34 \mu \mathrm{m}$ \\
\hline Segment time off-point & $U_{\text {time }}$ & $0.63 \mu \mathrm{m}$ \\
\hline Erosion rate & $E$ & $50.43 \frac{\mu \mathrm{m}}{\mathrm{khr}}$ \\
\hline Total rate uncertainty & $U_{E}$ & $14.85 \frac{\mu \mathrm{m}}{\mathrm{khr}}$ \\
\hline
\end{tabular}

\section{Alternative Methods}

Several alternative approaches to masked profilometry measurements exist. One method is making mass measurements on components before and after erosion segments. This method provides a globally averaged erosion rate, rather than a local rate. For the purpose of making design decisions, erosion based on a globally averaged measurement has the advantage of simplicity of comparison. But the globally averaged method may obscure underlying trends or local regions of high erosion. The 250 hour case study above was tracked with mass loss measurements. The component lost a mass of $0.25 \%$ during the erosion segment. The estimated mass loss based on a locally averaged erosion step of $12.5 \mu \mathrm{m}$ was $0.31 \%$. The general agreement of the two values demonstrates the feasibility of the method, although improvements should be implemented for improved accuracy. The discrepancy between the two values is likely due to a number of factors. For instance, the density of the component was assumed from vendor literature, the moisture content was not carefully controlled, the geometry of the component was estimated using nominal CAD models, and the predicted mass loss was based on only the 250 hour case study which includes an estimated 29\% uncertainty. But even with the assumptions of a mass loss based approach, operating at two different conditions and tracking mass loss provides a simpler decision mechanism for design and planning purposes than evaluating several locally varying profiles.

\section{Conclusion}

The measurement of erosion rates using non-contact profilometry and masked regions has been investigated to determine a conservative uncertainty of the approach described. A number of sources of uncertainty have been discussed and quantified using two case studies from the AEPS HERMeS thruster. The importance of carefully 
planning erosion test sequences should be highlighted by the potentially large uncertainty calculated for short test segments and low erosion rates. In the two cases investigated a long duration test resulted in a measured erosion rate uncertainty of $8.4 \%$ of measured value, and a short duration test resulted in an uncertainty of $29.4 \%$. The main contributing sources of uncertainty have been outlined and can provide insight to improved measurements.

\section{References}

[1] Smith, B. K., Nazario, M. L., and Cunningham, C. C., "Solar Electric Propulsion Vehicle Demonstration to Support Future Space Exploration Missions," Space Propulsion 2012, Bordeaux, France, 2012.

[2] Herman, D. A., Tofil, T., Santiago, W., Kamhawi, H., Polk, J. E., Snyder, J. S., Hofer, R. R., Picha, F., Jackson, J., and Allen, M., "Overview of the Development and Mission Application of the Advanced Electric Propulsion System (AEPS)," $35^{\text {th }}$ International Electric Propulsion Conference, IEPC Paper 2017-284, Electric Rocket Propulsion Society, Fairview Park, OH, 2017.

[3] Frieman, J. D., Kamhawi, K., Williams, G., Herman, D. A., Peterson P. Y., Gilland, J. H., and Hofer, R. R., "Long Duration Wear Test of the NASA HERMeS Hall Thruster," 54th AIAA/SAE/ASEE Joint Propulsion Conference, AIAA Paper 2018-4645, Cincinnati, OH, 2018.

[4] Ahern, D. M., Frieman, J. D., Williams, G., Mackey, J. A., Haag, T. W., Huang, W., Kamhawi, H., Herman, D., Peterson, P. Y., Gilland, J., and Hofer, R., "In-situ Diagnostic for Assessing Hall Thruster Wear," 54th AIAA/SAE/ASEE Joint Propulsion Conference, AIAA Paper 2018-4721, Cincinnati, OH, 2018.

[5] Yim, J., Soulas, G.C., Shastry, R., Choi, M., Mackey, J., and Sarver-Verhey, T.. "Update of the NEXT ion thruster service life assesment with post-test correlatio to the long duration test," IEPC Paper 2017-061.

[6] Figliola, R.S., and Beasley, D.E., "Theory and Design for Mechanical Measurements 3 ${ }^{\text {rd }}$," Wiley, 2014.

[7] Abernethy, R.B., Benedict, R.P., and Dowdell, R.B., "ASME Measurement Uncertainty," Journal of Fluids Engineering, Vol. 107, 1985.

[8] Moffat, R.J., , "Contributions to the Theory of Single-Sample Uncertainty Analysis," Transaction of the ASME, Vol. 104, 1982.

[9] Castrup, S. et. al., "Measurement Uncertainty Analysis Principles and Methods," National Aeronautics and Space Administration, NASA-HDBK-8739.19-3, 2010.

[10] Polk, J.E., Jaskowsky, W., Kelley, A.J., and Jahn, G., "Measurement of MPD Thruster Erosion Using Surface Layer Activation," J. Propulsion, Vol. 3, No. 1, (1987).

[11] ISO 5436-1 Geometrical Product Specifications (GPS) - Surface texture: Profile method; Measurement standards,” (2000).

[12] ANSI/NCSL Z540-2-1997 “U.S. Guide to the Expression of Uncertainty in Measurement”, (1997). 